\title{
Fisiologia da percepção do estresse salino em híbridos de tangerineira "Sunki Comum" sob solução hidropônica salinizada
}

Francisco Vanies da Silva Sá ${ }^{*}$, Marcos Eric Barbosa Brito², Luderlândio de Andrade Silva², Romulo Carantino Lucena Moreira², Pedro Dantas Fernandes ${ }^{3}$, Lizaiane Cardoso de Figueiredo²

'Universidade Federal Rural do Semi-Árido, Mossoró, RN, Brasil

\begin{abstract}
Resumo
Objetivou-se estudar a fisiologia da percepção e a tolerância ao estresse salino em híbridos de tangerineira 'Sunki Comum'. O experimento foi conduzido em ambiente protegido do Centro de Ciências e Tecnologia Agroalimentar, CCTA, da Universidade Federal de Campina Grande, UFCG, Pombal, PB, em delineamento experimental de blocos casualizados, com esquema fatorial (2x4), composto por dois níveis de salinidade $\left(0,3\right.$ e 4,0 dS $\left.\mathrm{m}^{-1}\right)$ e quatro híbridos de tangerineira 'Sunki Comum' (TSKC), a saber: 1-TSKC x Citrange argentina (CTARG) -019; 2- TSKC $\times$ Citrumelo Swingle (CTSW) -028; 3 - TSKC x CTSW-033 e 4 - TSKC x CTSW-041), com três repetições e quatro plantas por parcela. As plantas foram cultivadas em sistema hidropônicoe, aos 90 dias após a semeadurainiciou-se a aplicação das soluções nutritivas preparadas com águas salinizadas nos portas-enxertos, procedendo-se avaliações relativas às trocas gasosas e fluorescência da clorofila aàs 24 e 48 horas do iníciodo estresse salino. Nos híbridosTSKC x CTSW-028, TSKC x CTSW-033 e TSKC x CTSW-041 a submissão à solução salina durante as primeiras $24 \mathrm{~h}$ proporcionou alterações nos aspectos fisiológicos; no TSKC x CTARG - 019, a salinidade ocasionou alterações após 48h.Quanto ao grau de tolerância dos híbridos, pode-se classificar, em escala decrescente: TSKC x CTARG-019 $>$ TSKC $\times$ CTSW-028 = TSKC $\times$ CTSW-041 > TSKC $\times$ CTSW-033.
\end{abstract}

Palavras-Chave: Citrus sp., salinidade, tolerância

\section{Physiology of perception of saline stress in 'Common Sunki' mandarin hybrids under saline hydroponic solution}

\begin{abstract}
In order to study the physiology of perception of saline stress in 'Common Sunki' mandarin hybrids, an experiment was realized in a greenhouse at the CCTA - UFCG, Pombal, PB, Brazil, using using a randomized block design with a $2 \times 4$ factorial, with two levels of salinity $\left(0.3\right.$ and $\left.4.0 \mathrm{dS} \mathrm{m}^{-1}\right)$ and four 'Common Sunki' mandarin hybrids (1 - TSKC x CTARG-019; 2 - TSKC x CTSW-028; 3 - TSKC x CTSW-033 and 4 - TSKC $\times$ CTSW-041), with three replications and four plants per plot. The plants were grown hydroponically and 90 days after sowing, a saline water solution was applied on rootstocks and evaluations of gas exchange and chlorophyll fluorescence were carried out 24 and 48 hours after saline stress. Changes on physiologic conditions were observed in the hybrids TSKC x CTSW - 028, TSKC x CTSW - 033 and TSKC x CTSW - 041 during the first 24 hours of saline stress. Fot the hybrid TSKC x CTARG - 019, changes were observed after 48 hours od salinity stress. According to salinity tolerance, the hybrids could be classified as follows: TSKC $\times$ CTARG $-019>$ TSKC $\times$ CTSW $-028=$ TSKC x CTSW - 041 > TSKC x CTSW - 033.
\end{abstract}

Keywords: Citrus sp., salinity, tolerance 


\section{Introdução}

Os problemas de escassez de água em regiões áridas e semiáridas são frequentemente observados ao longo do globo terrestre, limitando os recursos hídricos para o consumo humano, impulsionando o uso de água de qualidade inferior na agricultura, a exemplo de salinas. Segundo Neves et al. (2009), é comum, na região semiárida brasileira, a utilização de fontes de água com alta concentração de sais, principalmente no que diz respeito aos sais de sódio, os quais proporcionam efeitos negativos sobre os solos e as plantas cultivadas. Comoconsequência, tem-se a perda da capacidade produtiva dos solos e enormes prejuízossocioeconômicos (Soares et al., 2012).

Tal perda da capacidade produtiva é variável com a tolerância à salinidade, que depende de muitos fatores, a saber: espécie, cultivar, estádio fenológico, características dos sais, intensidade e duração do estresse salino (Fernandes et al., 2011; Silva et al., 2012), conduzindo à busca do melhoramento genético para obtenção de materiais tolerantes. Sendo importante, todavia, que o material a ser melhorado tenha potencial produtivo e aceitabilidade no mercado, a exemplo dos citros.

Quanto aos citros, denota-se que a produção nas regiões semiáridas do nordeste brasileiro, é concentrada nos estados de Bahia, Sergipe e Paraíba, onde há sérios problemas com a salinidade no solo e na água, tendo em vista a sua sensibilidade as altas concentrações de sais, com uma salinidade limiar em torno de 2,0 dSm${ }^{-1}$ (Singh et al., 2003), em uma região onde comumente as águas disponíveis para uso na irrigação contém teores relativamente acentuados de sais, sendo encontrados frequentemente valores que chegam a 5,0 dS $\mathrm{m}^{-1}$ (Nobre et al., 2012; Soares et al., 2012).

Ademais, a citricultura brasileira encontra-se basicamente alicerçada no portaenxerto limoeiro 'Cravo' (CitruslimoniaOsbeck) (Mattos Junior et al., 2005), provendo uma baixa variabilidade genética aos pomares brasileiros, o que acarreta a suscetibilidade à fatores bióticos e abióticos, como o estresse salino (Mattos Junior et al., 2005).
Portanto, fazem-se necessárias atividades enérgicas para a seleção de materiais com potencial tolerância a estes fatores. Denota-se que algumas pesquisas tem evidenciado a seleção de alguns materiais com potencial tolerância, a exemplo dos trabalhos feitos por Brito et al. (2008), Fernandes et al. (2011), Brito et al. (2014) e Perez-Perez et al. (2015), estudando tanto materiais potenciais não enxertados quanto enxertados, o que, todavia, é um processo importante, mas que demanda tempo. Podendo-se ter indicativos de materiais com tolerância ainda na fase inicial de estresse, o que pode diminuir o rol de genótipos a serem avaliados por períodos mais extensos, a exemplo do que Silva et al., (2014), fizeram em híbridos trifoliados de citros, por meio de estudo de trocas gasosas e da fluorescência da clorofila $a$.

Com isso, objetivou-se estudar a fisiologia da percepção e a tolerância ao estresse salino em híbridos de tangerineira 'Sunki Comum'.

\section{Material e Métodos}

O experimento foi desenvolvido em ambiente protegido, no Centro de Ciências e Tecnologia Agroalimentar - CCTA da Universidade Federal de Campina Grande UFCG, localizado no município de Pombal, Paraíba, PB, nas coordenadas geográficas 6०47'20" de latitude $S$ e $37^{\circ} 48^{\prime} 01^{\prime \prime}$ de longitude W, a uma altitude média de 184 m; no período de 12 de fevereiro à 20 de julho de 2013.

Estudaramu-sedois níveis de salinidade da água [0,3 (água de abastecimento local) e 4,0 dSm${ }^{-1}$ (água de abastecimento acrescida de sais)]usadas nas preparações das soluções nutritivas aplicadas em quatrohíbridos de porta-enxertos de citros $\{\mathbf{1}$ - tangerineira Sunki(Citrussunki (Hayata) hort. exTanaka) Comum (TSKC) $\times$ citrange $[C$. sinensis $\times P$. trifoliata (L.) Raf.] Argentino (CTARG)-019; 2 TSKC x citrumelo 'Swingle' [C. paradisiMacfad. $x$ Poncirustrifoliata (L.) Raf.] (CTSW)-028; 3 - TSKC x CTSW-033; 4 - TSKC x CTSW-041\}.Tais tratamentos foram distribuídos em esquema fatorial $(2 \times 4)$ em um delineamento experimental de blocos ao acaso, com três repetições e quatro plantas úteis por parcela. Ressalta-se que as sementes dos híbridos mencionados foram obtidas junto 
ao Programa de Melhoramento Genético de Citros da Embrapa Mandioca e Fruticultura PMG Citros, Cruz das Almas-BA.

Os níveis de salinidade da água testados foram estabelecidos em razão da salinidade limiar de variedades de citros descrita por Singh et al. (2003) (2 dS $\left.\mathrm{m}^{-1}\right)$, sendo adotado um nível abaixo e outro acima desta.

As sementes, devidamente selecionadas e tratadas com fungicida disulfato de thiram $(4 \mathrm{~g}$ $\mathrm{kg}^{-1}$ de sementes), foram semeadas na razão de três por recipiente, sendo cobertas com o substrato. Os recipientes usados para cultivo das plantas de citros foram os vasos de Leonard adaptados com garrafas pet (Santos et al., 2009). A parte superior dos vasos foi preenchida com 1,5 L do substrato comercial à base de pó de coco de modo a garantir que não houvesse interferência de sais presentes no material, assim como disponibilidade de nutrientes, por se tratar de um substrato inerte. Na parte inferior permaneceramas soluções nutritivas sob fluxo contínuo, as quais seguiram as recomendações de Hoagland \& Arnon (1950), sendo, porém, acrescentado $25 \%$ de ferro EDTA, a fim de suprir as necessidades nutricionais dos híbridos (Tabela 1) observadas em ensaio preliminar. $O \mathrm{pH}$ da solução foi regulado para permanecer na faixa de 5,5 a 6,6, sendo este monitorado diariamente com pHmetro portátil, e nos casos de calibaração da solução utilizou-se as soluções de $\mathrm{KOH}$ a 0,1 $\mathrm{mol} \mathrm{L}^{-1} \mathrm{e} \mathrm{H}_{2} \mathrm{SO}_{4}$ a $0,1 \mathrm{~mol} \mathrm{~L}{ }^{-1}$.

Tabela 1. Concentração dos nutrientes na solução nutritiva para cultivo hidropônico de citros (adaptado de Hoagland\& Arnon, 1950).

\begin{tabular}{|c|c|c|c|c|c|c|c|c|c|c|c|c|}
\hline Nutrientes & $\mathrm{N}$ & $P$ & K & $\mathrm{Ca}$ & $\mathrm{Mg}$ & $S$ & $\mathrm{Fe}$ & $\mathrm{Mn}$ & $B$ & $\mathrm{Cu}$ & $\mathrm{Zn}$ & Mo \\
\hline & & & & & & & $\mathrm{mmol} \mathrm{L}$ & & & & & \\
\hline Concentração & 15 & 1 & 6 & 5 & 2 & 2 & 0,0625 & 0,01 & 0,05 & 0,003 & 0,0008 & 0,001 \\
\hline
\end{tabular}

Após preparação, os vasos de Leonard foram envolvidos com plástico dupla face, visando reduzir a evaporação da solução; ainda, cada vaso foi conectado, por meio de mangueira, a um reservatório contendo a solução nutritiva, que, por sua vez, possibilitava o abastecimento contínuo dos recipientes. O nível deste recipiente era verificado diariamente, em termos de volume e de condutividade elétrica das soluções, o que eram indicadores de incremento de solução ou de troca das mesmas, conforme o tratamento, garantindo-se que o substrato ficasse com umidade sempre próxima à da capacidade de contêiner.

Os seedlings foram repicados a fim de cultivar os clones de origem nucelar, cultivandose apenas um indivíduo por recipiente. Adotaram-se todos os cuidados de controle de ervas-daninhas e de prevenção e controle de pragas, recomendados na produção de mudas cítricas (Mattos Junior et al., 2005).

A água salinizada até $4,0 \mathrm{dS} \mathrm{m}^{-1}$, usada na mistura com a solução nutritiva, foi preparada de modo a se ter uma proporção equivalente de 7:2:1, entre $\mathrm{Na:Ca:Mg}$, sendo considerada, para tanto, a relação entre $C_{a}$ e concentração de sais $\left(10^{*} \mathrm{mmol}_{\mathrm{c}} \mathrm{L}^{-1}=1 \mathrm{dS} \mathrm{m}^{-1} \mathrm{de}\right.$
$\mathrm{CE}_{a}$ ), extraída de Rhoades et al. (1992), válida para $\mathrm{CE}_{\mathrm{a}}$ de 0,1 a 5,0 dS $\mathrm{m}^{-1}$ em que se enquadra o nível testado, tendo como base a água de abastecimento existente no local. Talrelação esta que reflete os íons predominantes em fontes de água utilizada para irrigação, em pequenas propriedades do Nordeste brasileiro (Medeiros et al., 2003). O preparo das soluções com as diferentes condutividades elétricas (CE) foi por adição dos sais a água até ser atingido o nível desejado de CE, conferindo-se os valores com um condutivímetro portátil ajustado para a temperatura de $25^{\circ} \mathrm{C}$.

$\mathrm{Na}$ água de abastecimento $\left(0,3 \mathrm{dS} \mathrm{m}^{-1}\right)$ e na água salinizada $\left(4,0 \mathrm{dS} \mathrm{m}^{-1}\right)$, se adicionou a solução nutritiva, que possuiauma condutividade elétrica de $2,3 \mathrm{dS} \mathrm{m}^{-1}$, resultando em soluções nutritivas de CE 2,6 e 6,3 dS $\mathrm{m}^{-1}$, respectivamente, que eram calibradas diariamente.

Aos 90 dias após a semeadura os híbridos de citros foram submetidos aos tratamentos salinose, às 24 e 48 horas depois, avaliados quanto ao estabelecimento fisiológico em relação ao estresse salino, mensurando-se a taxa de assimilação de $\mathrm{CO}_{2}(A)\left(\mu \mathrm{mol} \mathrm{m}{ }^{-2} \mathrm{~s}^{-1}\right)$, transpiração (E) $\left(\mathrm{mmol} \mathrm{de}_{2} \mathrm{O} \mathrm{m}^{-2} \mathrm{~s}^{-1}\right)$, condutância estomática (gs) (mol de $\mathrm{H}_{2} \mathrm{Om}^{-2} \mathrm{~s}^{-1}$ ) e a concentração interna 
de $\mathrm{CO}_{2}$ (Ci) na primeira folha madura contada a partir do ápice, utilizando-se o equipamento portátil de medição de fotossíntese "LCPro+" da ADC BioScientific Ltda. Com esses dados quantificaram-se a eficiência no uso da água (EUA) (A/T) $\left[\left(\mu \mathrm{mol} \mathrm{m} \mathrm{m}^{-2} \mathrm{~s}^{-1}\right)\left(\mathrm{mmol} \mathrm{H} \mathrm{O}_{2} \mathrm{~m}^{-2} \mathrm{~s}^{-1}\right)^{-1}\right]$ e a eficiência instantânea da carboxilação $\Phi_{C}$ (A) Ci) (Machado et al.,2007; Magalhães Filho et al., 2008). Nessas mesmas folhas foram colocadas pinças foliares, e após um período de 30 minutos de adaptação ao escuro, foram quantificadas a fluorescência inicial ( $\mathrm{Fo})$, a fluorescência máxima (Fm), a fluorescência variável (Fm-Fo) e a eficiência quântica do fotossistema II - PSII (Fv/Fm) (Mendonça et al., 2010) usando-se o equipamento PEA - Hansatech.

Os resultados obtidos foram avaliados mediante análise de variância pelo teste ' $F$ '. Nos casos de significância, foi realizado o teste de agrupamento de médias (Scott e Knott até $5 \%$ de probabilidade) para $\circ$ fator porta-enxerto $e$ teste de médias (Tukey até $5 \%$ de probabilidade) para os níveis de salinidade da água (Ferreira, 2011).

\section{Resultados e Discussão}

Após as primeiras 24 horas da aplicação dos tratamentos, observou-se efeito significativo da interação entre a salinidade e os híbridos estudados nas variáveis Ci, A, EUA e (A/Ci) e efeito isolados dos fatores salinidade e híbridos para Eegs (Tabelas 2 e 3). Estes dados são diferentes dos relatados por Silva et al. (2014), no que concerne ao efeito da salinidade para (Ci) durante as primeiras $24 \mathrm{~h}$ de submissão do estresse salino em híbridos trifoliados recomendados para porta-enxertos.

Já às 48 horas após exposição à salinidade, houve efeito significativo da interação em todas as variáveis estudadas, exceto para EUA (Tabela 2 e 3). Este fato pode estar relacionado ao ajuste das relações hídricas dos materiais em função do estresse salino, sendo que esta variável reflete a relação entre a transpiração e a assimilação de $\mathrm{CO}_{2}$ (Taiz \& Zaiger, 2013).

Tais resultados confirmam informações de Ayers \& Westcot (1999), Fernandes et al., (2011) e de Brito et al (2014), que destacam que a salinidade pode exercer efeitos diferenciados entre genótipos de uma mesma espécie, já que se notou efeito da interação entre os fatores neste trabalho.

Sob o menor nível de salinidade e nas primeiras 24 horas, os híbridos TSKC x CTARG019,TSKC $\times$ CTSW-041 e TSKC $\times$ CTSW-033, obtiveram os melhores resultados para $A$, com

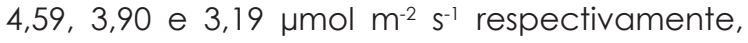
expressando maior potencial fisiológico na menor $\mathrm{CE}_{a^{\prime}}$ possivelmente relacionado às suas características de crescimento em relação ao híbrido TSKC x CTSW-028 que obteve 1,63 umol $\mathrm{m}^{-2} \mathrm{~s}^{-1}$ (Tabela 2).

Tabela 2. Teste de médias das variáveis concentração interna de $\mathrm{CO}_{2}(C i)$, transpiração $(E)$, condutância estomática (gs) e taxa de assimilação de $\mathrm{CO}_{2}$ (A) de híbridos de tangerina 'Sunki Comum' após 24 e 48 horas de imposição da salinidade da água em cultivo hidropônico.

\begin{tabular}{|c|c|c|c|c|c|c|c|c|}
\hline \multicolumn{5}{|c|}{$\mathrm{Ci}$} & \multicolumn{4}{|c|}{ gs } \\
\hline \multirow{2}{*}{ Híbridos de tangerineira } & \multicolumn{2}{|c|}{24 horas } & \multicolumn{2}{|c|}{ 48horas } & \multicolumn{2}{|c|}{24 horas } & \multicolumn{2}{|c|}{ 48horas } \\
\hline & $0,3\left(\mathrm{dS} \mathrm{m}^{-1}\right)$ & $4,0\left(\mathrm{dS} \mathrm{m}^{-1}\right)$ & 0,3 & 4,0 & 0,3 & 4,0 & 0,3 & 4,0 \\
\hline TSKCXCTARG - 019 & $284 \mathrm{aA}$ & 290 aA & $289 \mathrm{aA}$ & $263 a \mathrm{~A}$ & $0,10 \mathrm{aA}$ & $0,12 \mathrm{aA}$ & $0,08 \mathrm{aA}$ & $0,07 \mathrm{bA}$ \\
\hline TSKCxCTSW - 028 & $300 \mathrm{aA}$ & $261 \mathrm{aB}$ & $287 \mathrm{aA}$ & 276 aA & $0,04 \mathrm{bA}$ & $0,05 \mathrm{bA}$ & $0,06 \mathrm{bA}$ & $0,03 \mathrm{cB}$ \\
\hline TSKCXCTSW - 033 & $252 \mathrm{bA}$ & $269 \mathrm{aA}$ & 234 bB & 278 aA & $0,06 \mathrm{bA}$ & $0,07 \mathrm{bA}$ & $0,06 \mathrm{bA}$ & $0,03 \mathrm{cB}$ \\
\hline TSKCXCTSW - 041 & $265 \mathrm{bA}$ & $263 a A$ & $277 \mathrm{aA}$ & $279 \mathrm{aA}$ & $0,06 \mathrm{bA}$ & $0,06 \mathrm{bA}$ & $0,04 \mathrm{bB}$ & $0,11 \mathrm{aA}$ \\
\hline \multicolumn{5}{|l|}{ 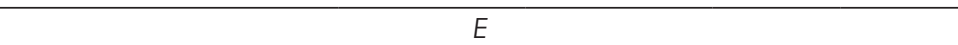 } & \multicolumn{4}{|c|}{$A$} \\
\hline \multirow{3}{*}{ HÍBRIDOS } & \multicolumn{4}{|c|}{ Exposição à salinidade (dS $\mathrm{m}^{-1}$ ) } & \multicolumn{4}{|c|}{ Exposição à salinidade (dS $\mathrm{m}^{-1}$ ) } \\
\hline & \multicolumn{2}{|c|}{24 horas } & \multicolumn{2}{|c|}{ 48horas } & \multicolumn{2}{|c|}{24 horas } & \multicolumn{2}{|c|}{ 48horas } \\
\hline & 0,3 & 4,0 & 0,3 & 4,0 & 0,3 & 4,0 & 0,3 & 4,0 \\
\hline TSKC $\times$ CTARG - 019 & $1,90 \mathrm{aA}$ & $2,23 \mathrm{aA}$ & $1,44 \mathrm{aA}$ & $1,52 \mathrm{aA}$ & $4,59 \mathrm{aA}$ & $4,57 \mathrm{aA}$ & 3,83 aA & $4,27 \mathrm{bA}$ \\
\hline TSKC $\times$ CTSW - 028 & $0,84 \mathrm{bA}$ & $1,21 \mathrm{bA}$ & $1,04 \mathrm{bA}$ & $0,73 \mathrm{bB}$ & $1,63 \mathrm{bB}$ & $3,47 \mathrm{aA}$ & $2,70 \mathrm{bA}$ & $2,01 \mathrm{cA}$ \\
\hline TSKC $\times$ CTSW - 033 & $1,24 \mathrm{bA}$ & $1,50 \mathrm{bA}$ & $1,26 \mathrm{aA}$ & $0,62 \mathrm{bB}$ & $3,90 \mathrm{aA}$ & $3,88 \mathrm{aA}$ & $4,72 \mathrm{aA}$ & $1,54 \mathrm{CB}$ \\
\hline TSKC $\times$ CTSW - 041 & $1,24 \mathrm{bA}$ & $1,35 \mathrm{bA}$ & $0,92 \mathrm{bB}$ & $1,88 \mathrm{aA}$ & $3,19 \mathrm{aA}$ & $3,42 \mathrm{aA}$ & $2,69 \mathrm{bB}$ & $5,34 \mathrm{aA}$ \\
\hline
\end{tabular}


Os resultados observados nos híbridosestão coerentes com a literatura onde são verificadas Aque variam de 3,0 a 9,0 $\mu \mathrm{mol} \mathrm{m}^{-2}$ $\mathrm{s}^{-1}$ (Machado et al., 2005; Machado et al., 2007; Magalhães Filho et al., 2008; López-Clement et al., 2011), exceto para híbrido TSKC x CTSW 028 que desempenhou um baixíssimo potencial fotossintético, que se procede também as 48 horas, onde o genótipo mesmo sob o menor nível de salinidade obteve valores deA inferior ao observado na literatura (Tabela 2).

Sob a aplicação dos tratamentos salinos nas primeiras 24 horas, apenas o híbrido TSKC $x$ CTSW-028 sofreu alterações significativas em suas trocas gasosas sob o maior nível de salinidade, reduzindo a $\mathrm{Ci}$ em $13 \%$ e ampliando em $112 \%$ a $A$, em $53,8 \%$ a EUA e em $160 \%$ a Фcquando comparado ao menor nível de salinidade (Tabelas 2 e 3).

Tal aumento pode ser relacionado ao aumento na produção fotossintatos, visando fugir das condições de estresse impostas pelo meio salino, promovendo a diluição de íons específicos na planta e, posteriormente, compartimentalização destes no vacúolo (Sá, 2014), o que resultou em efeitos positivos para o híbrido TSKC x CTSW - 028, já que se verificou a estabilização da taxa de assimilação e a Ci do mesmo após se passarem 48 horas de aplicação do tratamento salino (Tabela 2).

Em função das trocas gasosas serem reguladas pelos estômatos, a absorção de dióxido de carbono do meio externo promove perda de água, restringindo a entrada de $\mathrm{CO}_{2}$ (Machado et al., 2005) como observado pelas reduções na transpiração e estabilização da concentração interna de $\mathrm{CO}_{2}$ às 48 horas de exposição ao estresse (Tabela 2), podendo-se afirmar, com isso, que o híbrido TSKC x CTSW-028 apresenta além da capacidade de intensificar a produção de fotoassimilados e com isso aumentar eficiência de compartimentalização de sais no vacúolo (Sá, 2014). Ainda, deve-se destacar que esse fato ocorrendo com apenas 24 horas de início do estresse pode ser um sinal de maior sensibilidade deste híbrido.

Tabela 3. Teste de médias das variáveis eficiência no uso da água (EUA) e a eficiência instantânea da carboxilação(A/Ci) de híbrido de tangerina 'Sunki Comum' após 24 e 48 horas de imposição da salinidade da água em cultivo hidropônico.

\begin{tabular}{|c|c|c|c|c|}
\hline \multicolumn{5}{|c|}{ EUA } \\
\hline \multirow{3}{*}{ HÍBRIDOS } & \multicolumn{4}{|c|}{ Exposição a salinidade } \\
\hline & \multicolumn{2}{|c|}{24 horas } & \multicolumn{2}{|c|}{ 48horas } \\
\hline & $0,3 \mathrm{dSm}^{-1}$ & $4,0 \mathrm{dSm}^{-1}$ & $0,3 \mathrm{dSm}^{-1}$ & $4,0 \mathrm{dSm}^{-1}$ \\
\hline TSKC $\times$ CTARG - 019 & $2,5 \mathrm{aA}$ & $2,04 \mathrm{aA}$ & $2,72 \mathrm{aA}$ & $2,80 \mathrm{aA}$ \\
\hline TSKC x CTSW - 028 & $1,93 \mathrm{aB}$ & $2,94 \mathrm{aA}$ & $2,64 \mathrm{aA}$ & $2,69 \mathrm{aA}$ \\
\hline TSKC x CTSW - 033 & $3,18 \mathrm{aA}$ & $2,57 \mathrm{aA}$ & $3,75 \mathrm{aA}$ & $2,57 \mathrm{aA}$ \\
\hline TSKC $\times$ CTSW - 041 & $2,61 a A$ & $2,58 a A$ & $2,91 \mathrm{aA}$ & $2,86 a A$ \\
\hline \multicolumn{5}{|c|}{$\mathrm{A} / \mathrm{Ci}$} \\
\hline \multirow{3}{*}{ HÍBRIDOS } & \multicolumn{4}{|c|}{ Exposição a salinidade } \\
\hline & \multicolumn{2}{|c|}{24 horas } & \multicolumn{2}{|c|}{ 48horas } \\
\hline & $0,3 \mathrm{dSm}^{-1}$ & $4,0 \mathrm{dSm}^{-1}$ & $0,3 \mathrm{dSm}^{-1}$ & $4,0 \mathrm{dSm}^{-1}$ \\
\hline TSKC $\times$ CTARG - 019 & $0,016 a A$ & $0,015 \mathrm{aA}$ & $0,013 \mathrm{bA}$ & $0,016 \mathrm{aA}$ \\
\hline TSKC x CTSW - 028 & $0,005 \mathrm{bB}$ & $0,013 \mathrm{aA}$ & $0,009 \mathrm{bA}$ & $0,007 \mathrm{bA}$ \\
\hline TSKC x CTSW - 033 & $0,015 a \mathrm{~A}$ & $0,014 a A$ & $0,020 \mathrm{aA}$ & $0,005 \mathrm{bB}$ \\
\hline TSKC $\times$ CTSW - 041 & $0,012 a \mathrm{~A}$ & $0,016 \mathrm{aA}$ & $0,009 \mathrm{bB}$ & $0,019 \mathrm{aA}$ \\
\hline
\end{tabular}
significativa para os niveis de salinidade pelo teste de Tukey a $5 \%$ de probabilidade.
.

Nas 48 horas de exposição às soluções preparadas com águas salinas, contata-se efeito significativo da salinidade também nos híbridos TSKC x CTSW-033 e TSKC x CTSW-041, em adição as observadas no TSKC x CTSW - 028 (Tabelas 2 e 3), podendo-se inferir que tal sensibilidade seja inerente à características genéticas do cruzamento, já que não se observou diferenças no TSKC x CTAR - 019, todavia, estudos abrangendo um maior número de híbridos de um mesmo cruzamento deve ser realizado para dar maior embasamento a esta conclusão.

Nos híbridos da progênie TSKC x CTSW, numerados como 028, 033 e 041, as alterações ocorrentes às 48 horas foram notadas, principalmente, quanto a gs, E, A e na A/Ci; 
destacando-se redução na fotossíntese líquida na ordem $25,5 \%$ e de $67,3 \%$ nos genótipos TSKC $x$ CTSW - 028 e 033, respectivamente; já no híbrido TSKC x CTSW - 041 notou-se um incremento no valor da fotossíntese líquida de 98,5\% (Tabelas 3 e 4). Por outro lado, no híbrido TSKC x CTARG - 019 o aumento da salinidade ocasionou aumento da $A$, porém na ordem de $9,1 \%$, ou seja, uma pequena variação, permitindo deduzir que há maior estabilidade deste material sob condições de estresse salino, sendo um indicativo de maior tolerância.

Tabela 4. Teste de médias das variáveis fluorescência inicial, fluorescência máxima, fluorescência variável e eficiência quântica do fotossistema II doshíbridos de citros após 24 e 48 horas de imposição da salinidade da água em cultivo hidropônico.

\begin{tabular}{|c|c|c|c|c|c|c|c|c|}
\hline \multirow{4}{*}{ HÍBRIDOS } & \multicolumn{4}{|l|}{ Fo } & \multicolumn{4}{|c|}{$\mathrm{Fm}$} \\
\hline & \multicolumn{4}{|c|}{ Exposição à salinidade (dS $\mathrm{m}^{-1}$ ) } & \multicolumn{4}{|c|}{ Exposição à salinidade(dS $\left.\mathrm{m}^{-1}\right)$} \\
\hline & \multicolumn{2}{|c|}{24 horas } & \multicolumn{2}{|c|}{ 48horas } & \multicolumn{2}{|c|}{24 horas } & \multicolumn{2}{|c|}{ 48horas } \\
\hline & 0,3 & 4,0 & 0,3 & 4,0 & 0,3 & 4,0 & 0,3 & 4,0 \\
\hline TSKC $\times$ CTARG - 019 & $558 \mathrm{aA}$ & $525 \mathrm{aA}$ & $504 a A$ & 459 aA & $2421 \mathrm{aA}$ & $2200 \mathrm{aB}$ & $2274 a A$ & $2054 \mathrm{bA}$ \\
\hline TSKC x CTSW - 028 & $502 \mathrm{aA}$ & $475 \mathrm{aA}$ & $404 \mathrm{aA}$ & $459 \mathrm{aA}$ & $1899 \mathrm{CB}$ & $2188 \mathrm{aA}$ & $1576 \mathrm{bB}$ & $2194 \mathrm{aA}$ \\
\hline TSKC x CTSW - 033 & $414 \mathrm{bA}$ & $417 \mathrm{bA}$ & $434 \mathrm{aA}$ & $407 \mathrm{aA}$ & $2213 \mathrm{bA}$ & $2120 \mathrm{aA}$ & $2246 a A$ & $2268 \mathrm{aA}$ \\
\hline TSKC x CTSW - 041 & $446 \mathrm{aA}$ & 516 aA & $463 \mathrm{aA}$ & $471 \mathrm{aA}$ & $1958 \mathrm{cA}$ & $1837 \mathrm{bA}$ & 2076 aA & $1864 \mathrm{bA}$ \\
\hline \multicolumn{5}{|c|}{$\mathrm{Fv}$} & \multicolumn{4}{|c|}{ Eficiência quântica do fotossistema II } \\
\hline \multirow{3}{*}{ HÍBRIDOS } & \multicolumn{4}{|c|}{ Exposição à salinidade (dS $\left.\mathrm{m}^{-1}\right)$} & \multicolumn{4}{|c|}{ Exposição à salinidade $\left(\mathrm{dS} \mathrm{m}^{-1}\right)$} \\
\hline & \multicolumn{2}{|c|}{24 horas } & \multicolumn{2}{|c|}{ 48horas } & \multicolumn{2}{|c|}{24 horas } & \multicolumn{2}{|c|}{ 48horas } \\
\hline & 0,3 & 4,0 & 0,3 & 4,0 & 0,3 & 4,0 & 0,3 & 4,0 \\
\hline TSKC $\times$ CTARG - 019 & $1863 a A$ & $1675 \mathrm{aB}$ & $1770 \mathrm{aA}$ & $1594 \mathrm{bA}$ & $0,76 \mathrm{bA}$ & 0,76 aA & $0,77 \mathrm{bA}$ & $0,77 \mathrm{bA}$ \\
\hline TSKC $\times$ CTSW - 028 & $1397 \mathrm{bB}$ & $1713 a A$ & $1172 \mathrm{bB}$ & $1735 \mathrm{aA}$ & $0,73 \mathrm{CB}$ & $0,78 \mathrm{aA}$ & $0,74 \mathrm{bA}$ & $0,79 \mathrm{bA}$ \\
\hline TSKC $\times$ CTSW - 033 & $1799 \mathrm{aA}$ & $1703 \mathrm{aA}$ & $1812 \mathrm{aA}$ & $1861 \mathrm{aA}$ & $0,81 \mathrm{aA}$ & $0,80 \mathrm{aA}$ & $0,80 \mathrm{aA}$ & $0,82 \mathrm{aA}$ \\
\hline TSKC x CTSW - 041 & $1512 \mathrm{bA}$ & $1321 \mathrm{bB}$ & $1612 \mathrm{aA}$ & $1392 \mathrm{bA}$ & $0,77 \mathrm{bA}$ & $0,71 \mathrm{bB}$ & $0,77 \mathrm{bA}$ & $0,74 \mathrm{bA}$ \\
\hline
\end{tabular}

Para Baker \& Rosenqvit (2004), a redução da eficiência quântica do fotossistema Il é um indicativo de perturbações no sistema fotossintético causadas por estresses ambientais e bióticos, fato este confirmados pelos estudos de trocas gasosas no híbrido TSKC x CTSW-028, o qual intensificou sua atividade fotossintética após as 24 horas de exposição ao tratamento salino e estabilizou-se às 48 horas, em consonância com a eficiência quântica do fotossistema II (Tabelas 2,3 e 4).

A Fo não foi influenciada durante as primeiras 48 horas pelos níveis de salinidade impostos (Tabela 4), inferindo em ausência de danos no PSII, já que o aumento da fluorescência inicial indica destruição do centro de reação do PSII (P680) ou diminuição na capacidade de transferência da energia de excitação da antena ao PSII (Baker \& Rosenqvst, 2004). No
Às 24 horas após aplicação dos tratamentos foram observados efeitos significativos da interação para a variável Fm, Fve eficiência quântica do fotossistema II (Tabela 4). Observa-se ainda que após as 48 horas de submissão à salinidade, apenas as variáveis fluorescência máxima e fluorescência variável foram significativas para a interação, apontando a recuperação da eficiência da atividade do fotossistema ll após as 48 horas da aplicação do tratamento salino (Tabela 4). entanto, foram observados apenas efeitos diferenciados entre os híbridos estudados, sendo o híbrido TSKC $\times$ CTSW-033 que obteve os menores valores de fluorescência inicial, indicando o comprometimento da clorofila a (Tabela 4).

Assim, considerando que houve rendimentos satisfatórios de fluorescência nesses genótipos, pode-se dizer que os efeitos iniciais do estresse salino podem ser relativos a fatores estomáticos. Esse fato também foi verificado por Hussain et al. (2012), que relataram que as restrições na atividade estomática de plantas cítricas sob salinidade reduziram a taxa de assimilação líquida em função da redução da difusão de $\mathrm{CO}_{2}$ para o interior das folhas.

Quanto à fluorescência máxima dos híbridos de tangerina 'Sunki Comum', observase que TSKC $\times$ CTARG-019 e TSKC $\times$ CTSW-028 
sofreram alterações nesta variável, sendo constatadas reduções de 9,2\% para o híbrido TSKC x CTARG-019 nas primeiras 24 horas de submissão aos tratamentos, estabilizando-se depois de transcorridas 48 horas de estudo, interferindo no início da percepção do estresse salino por este híbrido, apesar de não terem sido verificadas alterações nas trocas gasosas desse híbrido (Tabela 2, 3 e 4).

Todavia, no híbrido TSKC $\times$ CTSW-028 constatou-se incremento na fluorescência máxima após a aplicação dos tratamentos de 13,3 e $39,2 \%$ às 24 e 48 horas, respectivamente (Tabela 4). A intensidade máxima de fluorescência $(\mathrm{Fm})$ denota o estado em que os centros de reações do PSIl atingiram sua capacidade máxima, evidenciando a condição reduzida de toda quinona (QA) pelos elétrons transferidos a partir do P680 (Baker \& Rosenqvst, 2004; Munns \& Tester, 2008), fato este que explica a intensa atividade de trocas gasosas do TSKC x CTSW - 028 após aplicação dos níveis de salinidade (Tabela 2).

Salienta-se ainda, que as reduções na fluorescência máxima o híbrido TSKC $x$ CTARG-019 não resultaram em efeito significativo da salinidade sob suas trocas gasosas (Tabela 2, 3 e 4).

Nos híbridos TSKC $\times$ CTARG-019, TSKC x CTSW-028 e TSKC x CTSW-041 ocorreram alterações na fluorescência variável durante as primeiras 24 horas após a imposição da salinidade, constatando-se reduções de 10,1 e 12,7\% para os híbridos TSKC x CTARG-019 e TSKC x CTSW-041,respectivamente e, um incremento de 22,6\% para o híbrido TSKC x CTSW-028 (Tabela 4).

Observa-se, ainda, que para TSKC $x$ CTSW-028 esse incremento se prolongou até às 48 horas de estudo,elevando em $48 \%$ a sua fluorescência variável,quando comparadas as duas situações estudadas. Enquanto que, para os outros dois híbridos observou-se uma estabilização da fluorescência variável às 48 horas de exposição à salinidade (Tabela 4).

Quanto à eficiência fotoquímica do Fotossistema II, apenas o híbrido TSKC x CTSW041 foi influenciado pela salinidade, obtendo um valor de $\mathrm{Fv} / \mathrm{Fm}(0,71)$ às 24 horas de exposição a nível $4,0 \mathrm{dSm}^{-1}$, sendo este $7,8 \%$ inferior ao obtido pelo menor nível de salinidade $(0,3 \mathrm{dSm}$ 1) (Tabela 6). Para Baker \& Rosenqvst (2004) a diminuição indica inibição da atividade fotoquímica, ressaltando-se que o valor de Fv/ Fm $(0,71)$ constatado no nível 4,0 dSm ${ }^{-1}$ ficou abaixo do índice 0,75 , considerado como limite para provocar dano no PSII (Silva et al., 2014), fato este que indica comprometimento do PSII, estimulando o mecanismo de tolerância do híbrido o qual teve sua atividade fotossintética estabilizada as 48 horas de exposição à salinidade.

\section{Conclusões}

Os híbridos são mais sensíveis às alterações na salinidade TSKC x CTSW-028, TSKC x CTSW-033 e TSKC x CTSW-041

O híbrido TSKC x CTARG-019 possui maior estabilidade nas tocas gasosas, mesmo apenas às $48 \mathrm{~h}$ sob salinidade.

A tolerância dos híbridos se comportou: TSKC $\times$ CTARG-019 $>$ TSKC $\times$ CTSW-028 $=$ TSKC $\times$ CTSW-041 > TSKC $\times$ CTSW-033.

O TSKC $\times$ CTSW-028 e TSKC $\times$ CTSW-041 desempenharam seus mecanismos de tolerância ao estresse salino durante as primeiras 24 horas de exposição ao estresse.

A salinidade comprometeu a atividade fotossintética do híbrido TSKC x CTSW - 033 durante as primeiras 48 horas de estresse salino, sendo este o mais sensível entre os híbridos estudados.

\section{Agradecimentos}

Ao CNPq e à Embrapa Mandioca e Fruticultura Tropical, pela parceria.

\section{Referências}

Ayers, R.S., Westcot, D.W. 1999. Qualidade da água na agricultura. Estudos FAO: Irrigação e Drenagem, 29. Campina Grande, Brasil. 153 p.

Baker, N.R.,Rosenqvst, E. 2004. Applications of chlorophyll fluorescence can improve crop production strategies: an examination of future possibilities. Journal of Experimental Botany 55: 1607-1621.

Brito, M.E.B., Soares, L.A.A., Fernandes, P.D., Lima, G.S., Sá, F.V.S., Melo, A.S. 2012. Comportamento fisiológico de combinações copa/porta-enxerto de citros sob estresse hídrico. Revista Brasileira de 
Ciências Agrárias 7: 857-865.

Brito, M.E.B., Fernades, P.D., Gheyi,H.R., Melo, A.S., Cardoso, J.A.F., Soares Filho, W.S.2008. Sensibilidade de variedades e híbridos de citrange à salinidade na formação de portaenxertos. Revista Brasileira de Ciências Agrárias 3: 343-353.

Brito, M.E.B., Fernandes, P.D., Gheyi, H.R., Melo, A.S., Soares Filho, W.S., Santos, R.T. 2014. Sensibilidade à salinidade de híbridos trifoliados e outros porta-enxertos de citros. Revista Caatinga 27:17-27.

Fernandes, P.D., Brito, M.E.B., Gheyi, H.R., Soares Filho, W.S., Melo, A.S., Carneiro, P.T. 2011. Crescimento de híbridos e variedades portaenxerto de citros sob salinidade. Acta Scientiarum Agronomy 33: 259-267.

Ferreira, D.F. 2011 . Sisvar: a computer statistical analysis system. Ciência Agrotecnologia 35: 1039-1042.

Hoagland, D.R., Arnon, D.I. 1950. The waterculture method for growing plants without soil. California Agricultural Experiment Station, Berkeley, EUA. 32 p.

Hussain, S.,Luro, F., Costantino, G.,Ollitrault, P.,Morillon, R. 2012. Physiological analysis of salt stress behavior of citrus species and genera: Low chloride accumulation as an indicator of salt tolerance. South African Journal of Botany 81: 103- 112.

López-Climent, M.F., Arbona, V., Pérez-Clemente, R.M., Gómez-Cadenas, A. 2011. Effects of cadmium on gas exchange and phytohormone contents in citrus. Biologia Plantarum 55: 187-190.

Machado, E.C., Oliveira, R.F., Ribeiro, R.V., Medina, C.L., Stuchi, E.S., Pavani, L.C. 2007. Deficiência hídrica agrava os sintomas fisiológicos da clorose variegada dos citros em laranjeira 'Natal'. Bragantia 66: 373-379.

Machado, E.C., Schmidt, P.T., Medina, C.L., Ribeiro, R.V.2005. Respostas da fotossíntese de três espécies de citros a fatores ambientais. Pesquisa Agropecuária Brasileira 40: 1161-1170.

Magalhães Filho, J.R.,Amaral, L.R., Machado, D.F.S.P., Medina, C.L., Machado, E.C. 2008. Deficiência hídrica, trocas gasosas e crescimento de raízes em laranjeira 'valência' sobre dois tipos de porta-enxerto. Bragantia67: 75-82.

Mattos Junior, D., Negri, J.D., Pio, R.S, Pompeu Junior, J. 2005.Citros. Instituto Agronômico e Fundag, Campinas, Brasil. 929p.

Medeiros, J.F., Lisboa, R.A., Oliveira, M., Silva Júnior, M.J., Alves, L.P. 2003. Caracterização das águas subterrâneas usadas para irrigação na área produtora de melão da Chapada do Apodi. Revista Brasileira Engenharia Agrícola e Ambiental7: 469-472.

Mendonça, A.V.R., Carneiro, J.G.A., Freitas, T.A.S., Barroso, D.G. 2010. Características fisiológicas de mudas de Eucalyptusspp submetidas a estresse salino. CiênciaFlorestal 20: 255-267.

Munns, R., Tester, M. 2008. Mechanisms of salinity tolerance. Annual Reviews of Plant Biology 59: 651-681.

Neves, A.L.R. Lacerda, C.F.,Guimarães, F.V.A., Hernandez, F.F.F., Silva, F.B.,Prisco, J.T., Ghey, H.R. 2009. Acumulação de biomassa e extração de nutrientes por plantas de feijão-de-corda irrigadas com água salina em diferentes estádios de desenvolvimento. Ciência Rural39:758-765.

Nobre, R.G., Lima, G.S., Gheyi, H,R., Medeiros, E.P., Soares, L.A.A., Alves, A.N. 2012. Teor de óleo e produtividade da mamoneira de acord $\square$ o com a adubação nitrogenada e irrigação com água salina. Pesquisa Agropecuária Brasileira 47: 991-999.

Perez-Perez, J.G., Garcia-Sanchez, F., Robles, J.M., Botía, P. 2015. 'Star Ruby' grapefruit and 'Clemenules' mandarin trees show different physiological and agronomic responses to irrigation with saline water. Irrigation Science 33: 191-204.

Sá, F.V.S. 2014. Fisiologia de porta-enxertos de citros sob água salina em cultivo hidropônico. 55 f. Monografia (Graduação em Agronomia). Universidade Federal de Campina Grande. Pombal, Brasil.

Silva, F.V., Soares, F.A.L., Gheyi, H.R., Travassos, K.D., Suassuna, J.F., Cardoso, J.A.F. 2012. Produção de citros irrigados com água moderadamente salina. Irriga 17: 396-407.

Silva, L.A., Brito, M.E.B., Sá, F.V.S., Moreira, R.C.L., Soares Filho, W.S., Fernandes, P.D. 2014. Mecanismos fisiológicos de percepção do estresse salino de híbridos de porta-enxertos citros em cultivo hidropônico. Revista Brasileira de Engenharia Agrícolae Ambiental 18:01-07.

Singh, A., Saini, M.L., Behl, R.K. 2003. Screening of citrus rootstocks for salt tolerance in semi-arid climates - A review. Tropics 13: 53-66.

Soares, L.A.A., Nobre, R.G., Gheyi, H.R., Lima, G.S., Silva, A.O., Soares, S.S. 2012. Componentes de crescimento da mamoneira cultivada com águas salinas e doses de nitrogênio. Irriga (Edição Especial) 40-54.

Taiz, L.,Zeiger, E. 2013. Fisiologia vegetal. 5.ed. Artmed,Porto Alegre, Brasil. 918 p. 